\title{
A VARIATIONAL APPROACH TO NUCLEAR MATTER WITH REALISTIC POTENTIALS
}

\author{
J. C. OWEN \\ Department of Theoretical Physics, Undoersity of Manchester, Manchester M13 9PL, UK \\ and \\ R. F. BISHOP and J. M. IRVINE \\ Department of Theoretical Phyolcs, Uniberdty of Manchester, Manchester M13 9PL, UK \\ and \\ Daresbury Laboratory, Sclence Renearch Councll, Warington WA4 4AD, UK
}

\section{Recoived S August 1976}

\begin{abstract}
Abatrect: A lowest order constrained variational method for calculating the bindins enerey of nuclear matter, provioully proposed by the prewent author, is extended to treat the strons tenior force components of realistic NN potentials. Numorical results are diven for three early potentials of Gammel, Chriatian and Thaler, and reaconable agreement is found with a provious calculation of Rintie. Ter Louw and Clark which included threo-body cluster contributions to the enerey. A range of five potentials edving $800 d$ flts to the experimental two-body NN date is slso studiod, and binding enerdes of typicklly $22 \mathrm{MoV}$ per nucleon at aturation denaities corresponding to $k_{5} \approx 1.6-1.7 \mathrm{fm}^{-1}$, are found. For three of the potentials considered, comparison is made with the recent results of Pandharipende and Wringe, which include the contributions to tho energy from all of the most significant many-body clusters, and excellent agreement is found. It is sureeted that explicit inchuilon of some of the neplected internal docress of froedom of the nucleoms, sweh as the poseibility of excitation to $\Delta(1236)$ states, might bring the equilibrium nuclear matter results closer to the empirical values.
\end{abstract}

\section{Introdaction}

In a recent paper ${ }^{1}$ ), hereafter referred to as I, we have described a method of lowest order constrained variation for the calculation of the ground-state energy of dense many-body systems. We have discussed in detail constraints which may be appliod to the two-body correlation function in the variational method of Jastrow correlation functions and which are so designed as to permit the truncation of the cluster series for the energy after two-body terms. In particular we have considerod a constraint in the form of the normalisation condition, and we have shown that by assuming a particular form for the long-range behaviour of the correlation function, we can perform an exact functional minimisation of the two-body energy with respect to the short-range behaviour of the correlation function. This functional minimisation procedure represents an enormous computational simplification over (unconstrained) methods that attempt to go beyond lowest order, and where it is necessary to paramotrize the short-range behaviour of the correlation function. In particular, it allows us to treat the partial wave dependence of realistic potentials with ease. 
In I we have compared the results of our lowest order constrained variational (LOCV) method, when applied to a range of semi-realistic central nucleon-nucleon potentials, with the results obtained by other authors using hypernetted chain methods to sum large classes of higher-order terms in the energy cluster series. For the potentials considered, we have found that a suitably constrained lowest order method is able to give results in substantial agreement with those obtained by hypernetted chain methods, for a wide range of densities up to and beyond nuclear matter density. In view of this success, we turn our attention in this work to a detailed consideration of more realistic nucleon-nucleon potentials, and in particular we focus upon potentials containing a strong tensor force component.

Ristig, Ter Louw and Clark ${ }^{2}$ ), hereafter referred to as RTC, have performed a variational calculation on three potentials of Gammel, Christian and Thaler ${ }^{3}$ ), in which they evaluate the energy up to and including three-body contributions in the cluster series for the energy. The three potentials considered are identical except in the triplet-even states, which contain a varying ratio of central to tensor force components. Since they do not also have the added complication of containing a spin-orbit or other non-central component apart from the tensor potential, they should serve as a good testing-ground for investigating the effect of the tensor force in the many-body problem. We calculate the ground-state energy for these three potentials using our LOCV method, suitably generalised to treat strong tensor forces. By comparison with the results of RTC, we find that our results are certainly consistent with the hope that a suitably constrained lowest order method is capable of giving good results up to and beyond nuclear matter density, even in the presence of a strong tensor force.

Thus motivated, we also present the results of our calculations for the ground-state energy of nuclear matter using five of the most realistic phenomenological internucleon potentials, viz. the super-soft (finite rectangular) core potential of Bressel, Kerman and Rouben ${ }^{4}$ ); the soft (Yukawa) core potential of Reid ${ }^{5}$ ) both in its original form, and with a correction to the ${ }^{1} S_{0}$ state potential as given by Kermode $e t$ al. ${ }^{6}$ ); and the (infinitely) hard core potentials of Reid ${ }^{5}$ ) and Hamada-Johnston ${ }^{7}$ ). Pandharipande and Wiringa ") have recently reported an extension of the fermion hypernetted chain method, which purports to include all of the important many-body cluster contributions and to correctly incorporate the tensor force. Using this method, they have given nuclear matter results for four internucleon potentials, three of which we also use. Direct comparison of our results with theirs is complicated by their use of different assumptions about the potential in states of total angular momentum $J>2$, and, more seriously, because their results are not entirely conclusive with respect to the saturation of the energy as the variational space is extended. However we find that, insofar as comparison can be made, the overall agreement between the two sets of results is most impressive.

Finally, we discuss the difficulties and uncertainties involved in the treatment of both the tensor force and the channel dependence of the realistic internucleon force in a lowest order approximation. It is concluded that there is every reason to believe that 
the method of lowest order constrained variation as described here is capable of giving good agreement for most physical potentials with the results of the more ambitious (and computationally much more difficult) schemes for incorporating higher-ordor clusters, up to and beyond nuclear matter density. We also discuss briefly the effect of incorporating one particular missing ingredient in the nuclear matter problem, namely the inclusion of the $\Delta(1236)$ resonance as an intermediate nucleon state. We conclude that the correct inclusion of this and other such "higher-order" processes could well bring our results into better agreement with the empirical values of the energy and saturation density of nuclear matter.

\section{Method}

We consider a system of $N$ nucleons of mass $m$ each contained in a volume $Q$ in the thermodynamic limit, where the number density is given in terms of the Fermi wavenumber $k_{F}$ as $\rho=N / Q=v k_{F}^{3} / 6 \pi^{2}$. The parameter $v$ is the spin-isospin degeneracy factor, equal to 4 or 2 for nuclear matter and neutron matter respectively. The nucleons are assumed to interact pairwise via a two-body potential $V(12)$. As in I we adopt a trial many-body wave function of the form,

$$
\boldsymbol{\Psi}=\boldsymbol{F} \boldsymbol{\Phi},
$$

where $\Phi$ is the (uncorrelated) ground-state wave function of $N$ independent nucleons, and $F=F(1 \ldots N)$ is an appropriate correlation operator. Specialising to the familiar Jastrow framework as in I yields the ground-state energy, $E$, of the interacting system, evaluated up to the two-body term in the cluster expansion, as ${ }^{2}$ ),

$$
\begin{gathered}
E / N=(3 / 10) \hbar^{2} k_{F}^{2} / m+E_{2} / N, \\
E_{2}=\sum_{i j}\langle i j|\mathscr{V}(12)| i j-j i\rangle, \\
\mathscr{V}(12) \equiv-\frac{1}{2} \hbar^{2} m^{-1}\left[f(12),\left[\nabla_{12}^{2}, f(12)\right]\right]+f(12) V(12) f(12),
\end{gathered}
$$

where $f(12)$ is the two-body Jastrow correlation operator which is assumed for its spatial dependence to be a function only of the relative coordinate $r_{12}$, and the Laplacian $\nabla_{12}^{2}$ acts on this same relative coordinate.

The two-body potentials that we consider have the general form,

$$
V(12)=\sum_{\alpha} V_{\alpha}(12) P_{\alpha}
$$

where $P_{\alpha} \equiv|\alpha\rangle\langle\alpha|$ is the $\alpha$-channel projection operator, and the two-body channel $\alpha \equiv(J S T)$ is defined in terms of the total angular momentum $J$ formed from the orbital and spin angular momenta $L$ and $S$ respectively, and the total isospin $T$ of the pair. Furthermore in each channel, we may write the potential in the form,

$$
V_{\alpha}(12)=V_{\alpha}^{\mathrm{C}}\left(r_{12}\right)+V_{\alpha}^{\mathrm{T}}\left(r_{12}\right) S_{12}+V_{\alpha}^{L S}\left(r_{12}\right) L \cdot S,
$$


where $S_{12} \equiv 3\left(\sigma_{1} \cdot P\right)\left(\sigma_{2} \cdot p\right)-\sigma_{1} \cdot \sigma_{2}$, and $L \cdot S$ are the usual tonsor and spin-orbit operators respectively. Clearly, for all states except the spin-triplet states with $L=$ $J \pm 1$, any tensor and spin-orbit components may be combinod with the central potential to form an effoctive central potential, which we continue to denote by $V_{a}^{C}$ in these channels.

By inserting a complete set of two-particle states $\left|r R, L S J M_{J} T M_{T}\right\rangle$ twice in the second of eqs. (2), where $r$ and $R$ are the relative and c.m. coordinates of the pair, and performing the trivial angular momentum algebra, we find

$$
\begin{array}{r}
E_{2}=\Omega^{-1} \sum_{\substack{k_{1} k_{2} \\
J S T L}} 4 \pi \int r^{2} d r j_{L}^{2}(k r) \mathscr{r}_{L L}^{J S T}(r)[T](2 J+1) \frac{1}{2}\left\{1-(-1)^{L+S+T}\right\}, \\
\mathscr{V}_{L L}^{J S T}(r) \equiv\langle L J S T|\mathscr{V}(r)| L J S T\rangle, \\
{[T] \equiv \begin{cases}(2 T+1), & v=4 \text { (nuclear matter) } \\
1, & v=2 \text { (neutron matter), }\end{cases} }
\end{array}
$$

where $k_{1}$ and $k_{2}$ are the wavenumbers of the two uncorrelated particles in the Fermi sea, $k=\frac{1}{2}\left(k_{1}-k_{2}\right)$, and $j_{L}$ is the usual spherical Bessel function. The summation over $T$ in eq. ( 5 ) is of course restricted to $T=1$ for neutron matter. For simplicity we assume, as has already been implied, that the correlation functions are momentum independent, thereby enabling the summation over $k_{1}$ and $k_{2}$ restricted to lie in the Fermi sphere of radius $k_{F}$, in 0 . (5), to be performed:

$$
\begin{gathered}
\frac{E_{2}}{N}=\frac{2 \pi \rho}{v^{2} k_{F}^{2}} \sum_{J S T L}[T](2 J+1) \frac{1}{2}\left\{1-(-1)^{L+8+T}\right\} \int_{0}^{\infty} \mathrm{d} x x^{2} \mathscr{V}_{L L}^{J S T}\left(k_{\mathrm{F}}^{-1} x\right) I_{L}(x), \\
I_{L}(x)=48 \int_{0}^{1} \mathrm{dzz}^{2}\left(1-\frac{3}{2} z+\frac{1}{2} z^{3}\right) j_{L}^{2}(x z) .
\end{gathered}
$$

In order to proceed further it is necessary to make an ansatz for the form of the correlation function $f(12)$. Corresponding to the form of the potential given in eq. (3), we use a generalisation of the form,

$$
f(12)=\sum_{\alpha} f_{\alpha}(12) P_{\alpha} .
$$

For uncoupled channels, in which the potential is effectively central, i.e. for spinsinglet, spin-triplet with $L=J$, and the ${ }^{3} P_{0}$ channels, we choose simply

$$
f_{a}(12)=f_{a}^{(1)}\left(r_{12}\right) \text {, }
$$

with no loss of generality. On the other hand, for the remaining channels, which are coupled in pairs $(\alpha, L=J \pm 1)$, there are four independent matrix elements $\left\langle\alpha, L=J \pm 1\left|f_{a}\right| \propto, L=J \pm 1\right\rangle$ whose number is reduced to three if we assume that $f(12)$ is hermitian. Accordingly, again with no loss of generality, we seek in this case a generalisation of $f_{a}(12)$ to the form of eq. (4), namely,

$$
f_{a}(12)=\sum_{i=1}^{3} f_{E}^{(l)}\left(r_{12}\right) P^{(l)} \text {, }
$$


where the operators $P^{(t)}$ are linear combinations of the operators $1, S_{12}$, and $L \cdot S$,

$$
P^{(i)}=a_{i}+b_{l} S_{12}+c_{i} L \cdot S .
$$

As usual the correlation function $f(12)$ is required to tend to unity as $r_{12} \rightarrow \infty$, and the natural extension of our method described in I for central potentials, is to require that this healing condition should further imply that $f^{(n)} \rightarrow 1$ soparately for each $(\alpha, i)$. This further restriction in turn implies that the operators $P^{(n)} ; i=1,2,3$ of eq. (13) should form a set of three mutually orthogonal projection operators in each $(\alpha, L)=$ $(T, J, S=1, L=J \pm 1)$ subspace,

$$
\left\{P^{(i)} P^{(n)}-\delta_{i j} P^{(i)}\right\}|T, J, S=1, L=J \pm 1\rangle=0 .
$$

It is easy to show that no sets of three such operators exist. One can, however, form an infinite number of pairs of such operators in the form,

$$
\begin{aligned}
P^{(2)} & =P, \quad P^{(3)}=1-P, \\
P & \equiv a+b S_{12}+c L \cdot S,
\end{aligned}
$$

where use of eqs. (14) and (15) yields that the coefficients $a, b$ and $c$ are any solutions of the two equations,

$$
\begin{aligned}
a^{2}+8 b^{2}+(J+2)(J-1) c^{2} & =a, \\
2 a-2 b-3 c & =1 .
\end{aligned}
$$

In this work the potentials have strong tensor force components, and we make the natural choice $c=0$ in eq. (15), which yields from eq. (16),

$$
P^{(2)}=\frac{2}{3}+\frac{1}{6} S_{12}=Q, \quad P^{(3)}=\frac{1}{3}-\frac{1}{6} S_{12}=1-Q .
$$

This choice corresponds to that made by both RTC and by the present authors in a previous publication ${ }^{9}$ ). An alternative simple choice is to take $b=0$, in which case we have

$$
P^{(2)}=P^{L-J-1}, \quad P^{(3)}=P^{L=J+1},
$$

where $P^{L-J \pm 1}$ are the projectors on to the $(\alpha, L=J \pm 1)$ subspaces. The choice (17) is clearly the more appropriate for the ${ }^{3} \mathrm{~S}_{1}-{ }^{3} \mathrm{D}_{1}$ states of nuclear matter, where the tensor force is strong and the spin-orbit force much weaker. However for the ${ }^{3} \mathbf{P}_{2}-{ }^{3} \mathbf{F}_{2}$ states this choice is by no means so obvious. We have investigated this numerically, as described later, and have found that eq. (17) is indeed the more appropriate choice in all cases. Accordingly in the coupled channels we use a correlation operator,

$$
f_{a}(12)=f_{a}^{(2)}\left(r_{12}\right) Q+f_{Q}^{(3)}\left(r_{12}\right)(1-Q) .
$$

The energy expression of eqs. (2), (6) and (8), with a correlation operator of the form given in eqs. (10), (11) and (19), is now readily evaluated by making use of the 
following operator identities:

$$
\begin{gathered}
\{Q, L \cdot S\}=2 P_{3}-3 Q+L \cdot S, \quad Q L \cdot S Q=-Q, \\
{\left[f,\left[\nabla^{2}, f\right]\right]=-2(\nabla f)^{2}, \quad\left[f,\left[\nabla^{2}, Q\right]\right]=0,} \\
{\left[Q,\left[L^{2}, Q\right]\right]=4 P_{3}-2 Q+2 L \cdot S,}
\end{gathered}
$$

where $f=f(r)$ is an arbitrary function, and $P_{3}=\frac{1}{4}\left(\sigma_{1} \cdot \sigma_{2}+3\right)$ is the projector on to spin-triplet states. The resulting final expression for the two-body energy may be written as,

$$
\frac{E_{2}}{N}=\frac{2 \pi \rho}{v^{2} k_{\mathrm{F}}^{2}} \frac{\hbar^{2}}{m}\left(\varepsilon_{c}+\varepsilon_{\mathrm{T}}\right)
$$

where $\varepsilon_{c}$ contains all of the contributions from the uncoupled states [i.e. spin-singlet, spin-triplet $(L=J)$, and the ${ }^{3} \mathrm{P}_{0}$ states], and is given by

$\varepsilon_{C}=\sum_{J S T L}[T](2 J+1) \frac{1}{2}\left\{1-(-1)^{L+s+T}, \int \mathrm{d} r\left\{f_{\alpha}^{(1)^{\prime 2}}+\frac{m}{\hbar^{2}} V_{\alpha}^{C} f_{\alpha}^{(1)^{2}}\right\} a_{\alpha}^{(1)^{2}}\left(k_{\mathrm{F}} r\right)\right.$,

where primes denote differentiation with respect to $r$, and where

$$
a_{\varepsilon}^{(1)^{2}}(x) \equiv x^{2} I_{L}(x)
$$

and $I_{L}(x)$ is given by eq. (9). The potential $V_{a}^{c}$ occurring in eq. (22) is the effective central potential for those uncoupled states, $V_{\varepsilon}^{c} \equiv\langle\alpha L|V(12)| \alpha L\rangle$. Thus, it may contain the diagonal contributions arising from any tensor or spin-orbit forces present in the original form of the potential $V(12)$, in the cases of the uncoupled spin-triplet $(L=J)$ and ${ }^{3} \mathrm{P}_{0}$ states. Similarly, the contribution $\varepsilon_{\mathrm{T}}$ to eq. (21) arises from the specifically tensor-coupled states [i.e. the spin-triplet $(L=J \pm 1)$ states], and is given by

$$
\begin{aligned}
\varepsilon_{\mathrm{T}} & =\sum_{J T}[T](2 J+1) \frac{1}{2}\left\{1-(-1)^{S+T}\right\} \int \mathrm{dr}\left[\left\{f_{\alpha}^{(2)^{\prime 2}}+m h^{-2}\left(V_{\alpha}^{\mathrm{C}}+2 V_{\alpha}^{\mathrm{T}}-V_{\alpha}^{L S}\right) f_{a}^{(2)^{2}}\right\}\right. \\
& \times a_{a}^{(2)^{2}}\left(k_{\mathrm{F}} r\right)+\left\{f_{a}^{(3)^{\prime 2}}+m \hbar^{-2}\left(V_{\alpha}^{\mathrm{c}}-4 V_{\alpha}^{\mathrm{T}}-2 V_{\alpha}^{L S}\right) f_{\alpha}^{(3)^{2}}\right\} a_{a}^{(3)^{2}}\left(k_{\mathrm{F}} r\right) \\
& \left.+\left\{r^{-2}\left(f_{a}^{(2)}-f_{\alpha}^{(3)}\right)^{2}+m \hbar^{-2} V_{\alpha}^{L S} f_{a}^{(2)} f_{\alpha}^{(3)}\right\} b_{a}^{2}\left(k_{\mathrm{F}} r\right)\right]
\end{aligned}
$$

where

$$
\begin{aligned}
a_{a}^{(2)^{2}}(x) & =x^{2}(2 J+1)^{-1}\left\{(J+1) I_{J-1}(x)+J I_{J+1}(x)\right\} \\
a_{\alpha}^{(3)^{2}}(x) & =x^{2}(2 J+1)^{-1}\left\{J I_{J-1}(x)+(J+1) I_{J+1}(x)\right\} \\
b_{\alpha}^{2}(x) & =x^{2} 2 J(J+1)(2 J+1)^{-1}\left\{I_{J-1}(x)-I_{J+1}(x)\right\} .
\end{aligned}
$$

The two-body energy expressed in eqs. (21)-(25) is now minimised with respect to variations in the functions $f_{\varepsilon}^{(i)}$, but subject to constraints which are fully discussed in $I$. In $I$, we have found that the constraints derived from the normalisation condition applied to the correlated two-body wave function, when used with central potentials, 
give results for the energy which are considerably more reliable than the results obtained using an alternate set of constraints, which we have termed boson constraints on account of their more direct applicability to Bose systems. Full details of our LOCV method which employs either the normalisation or boson constraints (and which we refer so as the LOCVN and LOCVB methods respectively) have been given in I. In a previous letter ${ }^{9}$ ) the LOCVB method has been applied to the Reid soft-core potential, and it is our intention in this work to apply the refinements of the LOCVN method to a range of realistic potentials, based on the success obtained by its use in I for a range of model potentials.

Suitably generalised to deal with non-central correlation functions of the form considered here, the normalisation constraints take the form of a certain bound on each of the components $f_{\varepsilon}^{(1)}(r)$, namely

$$
0 \leqq f_{\alpha}^{(t)}(r) \leqq h\left(k_{\mathrm{F}} r\right),
$$

together with an integral inequality of the form,

$$
1 \geqq k \equiv N^{-1} \sum_{i j}\left\langle i j\left|h^{2}-f^{2}(12)\right| i j-j i\right\rangle .
$$

The function $h(x) \geqq 0$, in eqs. (26) and (27), may be defined in terms of the functions $a_{a}^{(l) 2}(x)$ in eqs. (23) and (25), as

$$
\begin{aligned}
h^{-2}(x) & =(v x)^{-2} \sum_{a, i}[T](2 J+1) \frac{1}{2}\left\{1-(-1)^{L+S+T}\right\} a_{a}^{(l)^{2}}(x) \\
& =1-\frac{9}{v}\left[J_{1}(x) / x\right]^{2} .
\end{aligned}
$$

Eq. (27) is conveniently re-written in the form,

$$
\begin{array}{r}
1 \geqq k=4 \pi \rho\left(v k_{\mathrm{F}}\right)^{-2} \sum_{a, t}[T](2 J+1) \frac{1}{2}\left\{1-(-1)^{L+S+T}\right\} \int_{0}^{\infty} \mathrm{d} r\left\{h^{2}\left(k_{\mathrm{F}} r\right)-f_{\alpha}^{(t)^{2}}(r)\right\} \\
\times a_{\varepsilon}^{(l)^{2}}\left(k_{\mathrm{F}} r\right) .
\end{array}
$$

Minimisation of the two-body energy given by eqs. (21)-(25), subject to constraints (26) and (27) is effected as usual by solving the following set of Euler-Lagrange equations:

$$
g_{\varepsilon}^{(1)^{n}}=\left[a_{a}^{(1) "} \mid a_{a}^{(1)}+m \hbar^{-2}\left(V_{a}^{c}+\lambda\right)\right] \theta_{a}^{(1)}=0,
$$

arising from the uncoupled channels; and the coupled pairs of equations,

$$
\begin{aligned}
& g_{a}^{(2)^{*}}=\left[a_{a}^{(2)} / a_{a}^{(2)}+m \hbar^{-2}\left(V_{a}^{\mathrm{c}}+2 V_{\alpha}^{\mathrm{T}}-V_{\alpha}^{L S}+\lambda\right)+r^{-2} b_{\alpha}^{2} / a_{a}^{(2)}\right] g_{a}^{(2)} \\
& +\left(r^{-2}-\frac{1}{2} m h^{-2} V_{\alpha}^{L S}\right) b_{a}^{2}\left\{a_{a}^{(2)} a_{a}^{(3)}\right\}^{-1} g_{\alpha}^{(3)}=0, \\
& g_{a}^{(3)^{n}}=\left[a_{\alpha}^{(3)^{*}} / a_{a}^{(3)}+m h^{-2}\left(V_{a}^{\mathrm{C}}-4 a_{a}^{\mathrm{T}}-2 V_{\alpha}^{L S}+\lambda\right)+r^{-2} b_{a}^{2} / a_{a}^{(3)}\right] g_{a}^{(3)} \\
& +\left(r^{-2}-\frac{1}{2} m h^{-2} V_{\alpha}^{L S}\right) b_{\alpha}^{2}\left\{a_{\alpha}^{(2)} a_{\alpha}^{(3)}\right\}^{-1} g_{\alpha}^{(2)}=0,
\end{aligned}
$$


arising from the coupled channels, and where

$$
g_{a}^{(l)}(r) \equiv a_{a}^{(l)}\left(k_{F} r\right) f_{a}^{(l)}(r) .
$$

The primes in eqs. (30) and (31) denote differentiation with respect to $r$, and $\lambda$ is the Lagrange multiplier associated with constraint (27). Constraint (26) is incorporated by solving the Euler-Lagrange eqs. (30)-(32) only out to certain distances $d_{a}^{(l)}$, and setting $f_{\varepsilon}^{(t)}(r) \equiv h\left(k_{r} r\right)$ for $r \geq d_{k}^{(t)}$. We have previously shown $\left.{ }^{10}\right)$ that, for central potentials, $d_{\varepsilon}^{(l)}$ is the point where $f_{a}^{(n) \prime}=h^{\prime}$; and for the uncoupled states this remains true and unambiguous. For the coupled states we solve eqs. (31) as a pair of coupled equations for $f_{e}^{(2)}$ and $f_{\varepsilon}^{(3)}$ out to the point $d_{\varepsilon}^{(<)}$where either of the two correlation functions first has slope equal to that of the function $h$. We denote this function by $f_{\varepsilon}^{(<)}$, and hence $f_{\varepsilon}^{(<)}\left(d_{\varepsilon}^{(<)}\right)=h^{\prime}\left(k_{\mathrm{F}} d_{\varepsilon}^{(<)}\right)$. The remaining function of the pair is denoted by $f_{a}^{(>)}$, and for $r>d_{a}^{(<)}$, the relevant equation of the pair (32) for $f_{a}^{(>)}$ becomes decoupled, since $f_{\varepsilon}^{(<)} \equiv h$ for $r>d_{\varepsilon}^{(<)}$. It is advisable to solve this remaining equation for $f_{a}^{(>)}$out to well beyond the first point $d_{a}^{(>)}\left(>d_{a}^{(<)}\right)$where $f_{a}^{(>)^{\prime}}=h^{\prime}$. This is necessary in order to guard against a possible "switchback" behaviour in $f_{a}^{(>)}$, where a third point $d_{a}^{(>)}$where $f_{a}^{(>)}=h^{\prime}$ (i.e. a second maximum), might also exist, and out to which constraint (26) is satisfied. Such behaviour could arise due to what was in eq. (32) the coupling term [to $f_{k}^{(<)}$, now set equal to $h$ ], and is now an effective driving term. If this switchback behaviour does arise, then we choose $d_{a}^{(>)}$to be the greatest possible value in accord with the above prescription. In practice this is seldom a problem, and we find little evidence for such switchback behaviour except at very low densities. The proof that this is the correct minimisation procedure follows in a similar way as for the uncoupled states ${ }^{10}$ ).

The boundary conditions for eqs. (30)-(32), namely that the solutions for $g_{k}^{(l)}$ should be regular at the origin in the case of soft-core potontials and zero at the core radius for hard-core potentials, and that $f_{k}^{(i)}=h$ at $r=d_{k}^{(1)}$, then provide a unique solution. To obtain the full solution, the Lagrange multiplier $\lambda$ is first placed to zero, and if a solution $f_{a}^{(1)}$ exists in each channel with this choice, and if these satisfy constraint (29) as an inequality, then this is taken to be the solution. Otherwise, $\lambda$ is varied iteratively, solving the complete set of eqs. (30)-(32) at each iteration, until constraint (29) is satisfied as an equality.

\section{Reoults for the Gammel-Chriatian-Thaler potentinls}

Gammel, Christian and Thaler ${ }^{3}$ ) have given three different potential representations of the internucleon force, each of the form,

$$
V(r)=\sum_{i}\left[V_{i}^{\mathrm{C}}(r)+V_{i}^{\mathrm{T}}(r) S_{12}\right]
$$

where the suffix $i=(2 S+1, \pi)$ labels the spin and parity $\pi=(-1)^{L}$ of the various two- 
body channels. Both the central and tensor components in each channel have Yukawa form outside a state-independent hard-core:

$$
V_{l}(r)= \begin{cases}\infty, & r \leqq 0.5 \mathrm{fm} \\ A_{t} e^{-\mu_{r} r} /\left(\mu_{t} r\right), & r>0.5 \mathrm{fm} .\end{cases}
$$

The three potentials, labelled GCT, 5200 and 5100, are the same in all states except for the triplet-even states, where they have rospectively an increasing ratio of tensor to central components. The values for the various parameters $A$ and $\mu$ are given in table 1. Each of these potentials provides a good fit to low-energy two-nucleon scattering data and to the ground-state properties of the deuteron. They cannot be expected to give satisfactory fits to the high-energy data, since they do not contain spin-orbit or other higher-order terms, and hence they cannot be regarded in the same light as the "realistic" potentials considered in sect. 4. The very lack of a spin-orbit component in

TABLB 1

The parametere for the threo potentials labelled GCT, 5200 , and 5100

\begin{tabular}{|c|c|c|c|c|c|}
\hline State & & $\underset{\mathrm{MeV}}{A \mathrm{c}}$ & $\begin{array}{c}\mu_{c} \\
\left(\mathrm{~m}^{-1}\right)\end{array}$ & $\begin{array}{c}A_{\mathrm{T}} \\
(\mathrm{MoV})\end{array}$ & $\underset{\left(\mathbf{m}^{-1}\right)}{\boldsymbol{M}_{\boldsymbol{r}}}$ \\
\hline $\begin{array}{l}\text { singlet-odd } \\
\text { triplet-odd } \\
\text { singlet-oven } \\
\text { triplet-oven }\end{array}$ & $\begin{array}{l}G C T \\
5200 \\
5100\end{array}$ & $\begin{array}{l}113 \\
-150 \\
-905.6 \\
-6395 \\
-1587.7 \\
-121.94\end{array}$ & $\begin{array}{l}1.0 \\
1.5 \\
1.7 \\
2.936 \\
2.3576 \\
1.4112\end{array}$ & $\begin{array}{c}57.5 \\
-45 \\
-238.38 \\
-498.73\end{array}$ & $\begin{array}{l}1.12 \\
\\
0.73421 \\
1.1788 \\
1.4112\end{array}$ \\
\hline
\end{tabular}

The values in the singlet and triplet-odd states are common to all three. (The subucripts.C and T denote central and tensor components, respectively.)

these potentials does, however, provide us with a good testing-ground for our mothod, since it is clear that in this case we should use correlation function operators of the form (17) (i.e. pure tensor correlations), thereby simplifying the interpretation of the results.

The fact that the potentials depend explicitly only on the spin and parity of the twobody channel, and not on the total angular momentum, suggests that it is not necessary to exploit the full flexibility of using different correlation functions in each different (JST) channel, as described in sect. 2 . Instead, we average over any $J$-dependence, and assume correlation function operators of the form,

$$
f(r)=\sum_{z}\left\{f_{z}^{(1)}(r) P_{1}+\left[f_{z}^{(2)}(r) Q+f_{z}^{(3)}(r)(1-Q)\right] P_{3}\right\} P_{z}
$$

where $P_{1}$ and $P_{3}$ are the spin-singlet and spin-triplet projectors respectively, and $P_{n}(\pi= \pm)$ is the projector on to even or odd parity statos respectively. With this restricted set of correlation functions, it is possible to perform a similar analysis to that 
leading to eqs. (21) - (25), to get in this case,

$$
\frac{E_{2}}{N}=\frac{2 \pi \rho}{v^{2} k_{F}^{2}} \frac{\hbar^{2}}{m}\left(\varepsilon_{1}+\varepsilon_{3}\right)
$$

where $\varepsilon_{1}$ and $\varepsilon_{3}$ are now the contributions arising from the spin-singlet and spintriplet states respectively. These may in turn be expressed as,

$$
\begin{aligned}
\varepsilon_{1}= & \sum_{z}\left(u \delta_{z+}+v \delta_{z-}\right) \int \mathrm{d} r\left\{f_{z}^{(1)^{\prime 2}}+m \hbar^{-2} V_{1 z}^{\mathrm{C}} f_{z}^{(1)^{2}}\right\} a_{z}^{2}\left(k_{\mathrm{F}} r\right), \\
\varepsilon_{3}= & \sum_{z}\left(\nu \delta_{z+}+u \delta_{z-}\right) \int \mathrm{d} r\left[2 f_{z}^{(2)^{\prime 2}}+f_{z}^{(3)^{\prime 2}}\right. \\
& \left.+m h^{-2}\left\{2 f_{z}^{(2)^{2}}\left(V_{3 x}^{\mathrm{C}}+2 V_{3 \pi}^{\mathrm{T}}\right)+f_{z}^{(3)^{2}}\left(V_{3 z}^{\mathrm{C}}-4 V_{3 z}^{\mathrm{T}}\right)\right\}+4 r^{-2}\left(f_{z}^{(2)}-f_{z}^{(3)}\right)^{2}\right] a_{z}^{2}\left(k_{F} r\right),
\end{aligned}
$$

where the constants $u$ and $v$ are given by

$$
(u, v)= \begin{cases}(3,1), & v=4 \text { (nuclear matter) } \\ (1,0), & v=2 \text { (neutron matter). }\end{cases}
$$

The factors $\delta_{z+}$ and $\delta_{z-}$ in eqs. (37) and (38) are the usual Kronecker delta functions, and the functions $a_{z}^{2}$ are given by

$$
a_{ \pm}^{2}(x)=x^{2} \pm 9 j_{1}^{2}(x) \text {. }
$$

A similar summation is readily performed for the constraint (27), and minimisation of the two-body energy of eqs. (36)-(40) subject to constraints (26) and (27) gives for each parity an uncoupled equation and a coupled pair of equations similar to eqs. (30) and (31).

We have investigated the effect on the energy of restricting the correlation functions to depend only on the spin and parity of a state, and not on its total angular momentum, by allowing different correlation functions in each total angular momentum state $J$ for $0 \leqq J \leqq J_{\max }$, and then summing over all $J>J_{\max }$ as above. Wo find, for example, that with potential 5100 , and taking $J_{\max }=2$, we lower the energy of nuclear matter at a Fermi wavenumber of $k_{\mathrm{F}}=1.4 \mathrm{fm}^{-1}$ by only $0.4 \mathrm{MeV}$ as compared with simply summing $J$ from zero to infinity as above.

The three potentials considered here have also been studied in the context of nuclear matter by Ristig, Ter Louw and Clark ${ }^{2}$ ) (RTC). These authors also use correlation functions of the form given in eq. (35), but they parametrize the functions $f_{z}^{(l)}$ with simple assumed analytic forms. The two-body cluster contribution to the ground-state energy is then minimised with respect to these parameters. Furthermore, they then use the correlation functions so obtained to evaluate the three-body cluster contribution to the energy. RTC also perform a similar but simpler calculation where tensor correlations are excluded by setting $f_{z}^{(2)}=f_{z}^{(3)}$. Before discussing our own results, we make several comments on the method used and the results obtained by RTC. 


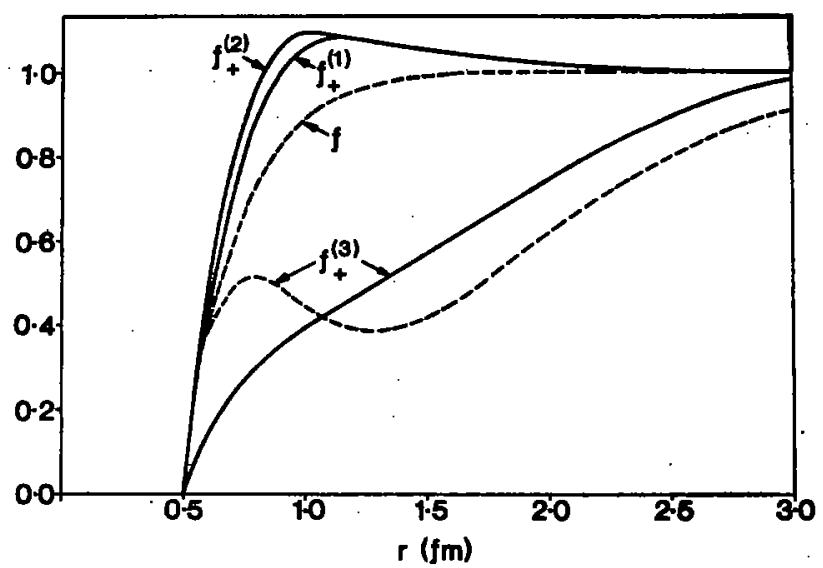

Fig. 1. The three even-state nuclear matter correlation functions for the potential 5100 , at $k_{z}=1.4$ fin $^{-1}$. The eolid and dahed curves are from the present work and from ref. ${ }^{2}$ ) roupectivoly. Note that in the latter work the restriction $f_{+}{ }^{(1)}=f_{+}{ }^{(2)} \equiv f$, has been made.

First, we note that in the triplet-even state the effective potential associated with the correlation function $f_{+}^{(3)}$, namely $V_{+}^{\mathrm{C}}-4 V_{+}^{\mathrm{T}}$, is always repulsive, even for large separations $r$. This means that in an unconstrained variation of the two-body cluster for the energy with respect to the parametrized function $f_{+}^{(3)}$, it is only the assumed functional form for $f_{+}^{(3)}$ which makes it heal to unity at large distances at all. This observation is reflected in the extremely long-ranged correlation functions $f_{+}^{(3)}$ which RTC obtain (and see fig. 1). A similar remark also applies to $f_{-}^{(2)}$, where the effective potential $\left(V_{-}^{\mathrm{c}}+2 V_{-}^{\mathrm{T}}\right)$ is also wholly repulsive. This defect shows up most strikingly for the GCT potential, where RTC find that when they have evaluated the cluster series up to and including three-body terms, with the correlation functions which minimise the two-body energy, then for Fermi momenta $\gtrsim 1.4 \mathrm{fm}^{-1}$ the more restrictive correlation function operators without tensor correlations actually yield more binding than the ones which include tensor correlations. If one accepts that at these densities the four-body and higher cluster contributions to the energy are small, it is clear that this indicates that the energies obtained by RTC with their parametrized correlation functions are rather higher than the true energies.

Secondly, we note that RTC find a fairly marked "switchback" effect of the typo referred to in sect. 2, in some of their tensor correlation functions. In fig.1 we show the even-state correlation functions which we obtain for the potential 5100 at $k_{\mathrm{F}}=1.4$ $\mathrm{fm}^{-1}$, and these are compared with those obtained by RTC. It is clear that our less restrictive (but constrained) minimisation of the two-body energy gives correlation functions which do not exhibit this "switchback" structure, whereas the function $f_{+}^{(3)}$ of RTC clearly does. This suggests that the effect is due largely to the simple parametrization of the correlation functions used by RTC, which in turn suggests again that more versatile correlation functions could give lower energies. The belief 
that the "switchback" behaviour is in large measure due to using overly restrictive parametrizations is strengthened by the following observation. As is clearly seen in fig. 1, the particular parametrizations for the even-state correlation functions used by RTC force them all to have equal slope at the core radius. However, the effective potentials in the channels associated with $f_{+}^{(1,2)}$ are both attractive outside the core, and minimisation of the two-body energy clearly demands that these correlation functions should heal rapidly to unity. What seems to be happening in the channel associated with $f_{+}^{(3)}$ is that this correlation function first gets "dragged" up with the other two, due to the parametrization, and then "switches back" as soon as it becomes free, only to rise again finally to heal due to the parametrization. These observations strengthen our belief that parametric minimisations, compared in this respect at least with a functional minimisation of the form considered by us, must be performed with extreme caution.

The arguments presented above suggest strongly that if RTC had minimised the energy evaluated up to three-body terms, rather than evaluated up to only two-body terms, and if they had used more versatile correlation functions, then they might well have obtained rather lower energies and considerably smaller three-body contributions.

In fig. 2 we show the energy-density curves obtained for nuclear matter in the present method by minimising the two-body energy of eqs. (36) to (40), subject to the

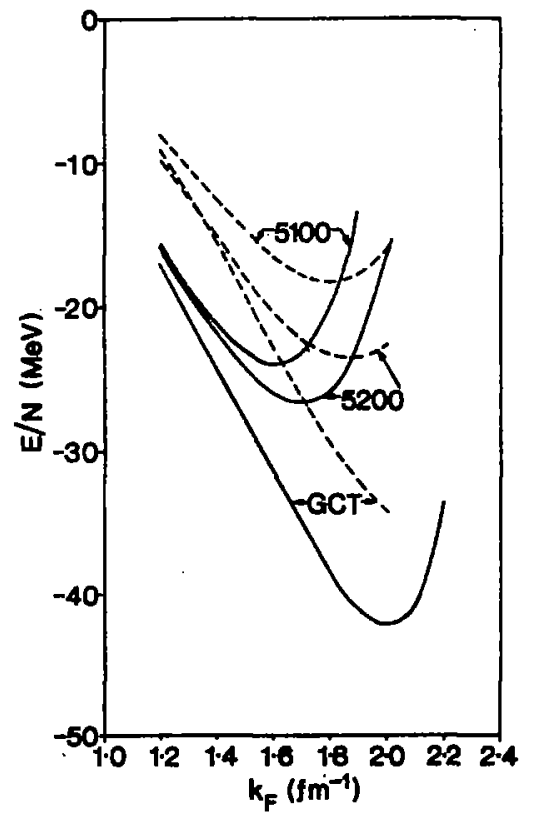

Fig. 2. The energy per nucleon of nuclear matter for the three potentials GCT, 5200 and 5100 . The solid curves are the results of the present work, and the dashed curves are taken from ref. ${ }^{2}$ ), including the threo-body cluster contributions, as explained in the text. 
normalisation constraints (26) and (27), for the three potentials GCT, 5200, and 5100. We also show the results of RTC $^{2}$ ) for the energy evaluated up to three-body clusters (but with the minimisation performed at the two-body level) as explained above. For the potentials 5200 and 5100 we show their curves $B(3)$ which include tensor correlations, whereas for the potential GCT we show their curve $A(3)$ which does not include tensor correlations, since this lies below their curve $B(3)$ in this case for the densities of interest, as indicated above.

The results shown in fig. 2 are clearly most encouraging. We have moved from a situation where RTC found no saturation at all of their two-body energy with respect to density, to a situation where out suitably constrained two-body energy-density curves are close to what we might crudely predict to be the exact results, bearing in mind the threo-body RTC results shown and the deficiencies of their minimisation scheme already noted. Our results are again clearly consistent with the hope that a suitably constrained lowest order method is capable of giving energies quite close to the exact binding energies, even for potentials with strong tensor components. Accordingly, in the next section, we present our nuclear matter results for a range of realistic NN potentials.

\section{Resalts for realistic potentials}

We turn now to potentials which provide accurate fits to the experimental two-body data. We consider the Yukawa soft-core potential of Reid ${ }^{5}$ ) (RSC); the RSC potential but with a correction to the ${ }^{1} \mathrm{~S}_{0}$ state interaction as given by Kermode and McKerrell ${ }^{6}$ ) (RSC +KM)'; the hard-core potentials of Reid ${ }^{5}$ ) (RHC), and of Hamada and Johnston ${ }^{7}$ ) (HJ); and the supersoft finite rectangular core potential of Bressel, Kerman and Rouben ${ }^{4}$ ) (BKR). The Reid ${ }^{5}$ ) potentials are only explicitly given for states with $J \leqq 2$, and for the sake of more direct comparison between the potentials, and because the other potentials give accurate fits to the scattering data only in the low-J states, we perform all our calculations on the assumption that each of the above five potentials has zero interaction in states with $J>2$.

The results of our nuclear matter calculations using the method outlined in sect. 2 are shown in fig. 3 , and a channel by channel breakdown of the various contributions to the energy per particle over a range of densities is given in tables 2-6 for each potential studied. These calculations were all performod using independent correlation functions in each (JST) state, and employing tensor correlations of the form (19) in both the triplet-even $\left({ }^{3} S_{1}-{ }^{3} D_{1}\right)$ and triplet-odd $\left({ }^{3} P_{2}-{ }^{3} F_{2}\right)$. states included. An example of the typical correlation functions obtained is shown in fig. 4.

As mentioned in sect. 2, we have tested for the effect of not specifically including spin-orbit correlations in the triplet-odd states by switching off the tensor correlations, and using either no spin-orbit correlations (i.e. the same central correlation function in both the ${ }^{3} \mathrm{P}_{2}$ and ${ }^{3} \mathbf{P}_{2}$ states) or an explicit spin-orbit correlation of the form (18)

t Wo have used potential 2 of table 1 of ref. ${ }^{6}$ ) for pp and $n n{ }^{1} S_{0}$ states, and the potential $C M=8$ of tablo 8 of ref. ${ }^{6}$ ) for $\mathrm{np}{ }^{2} \mathrm{~S}_{0}$ states. 


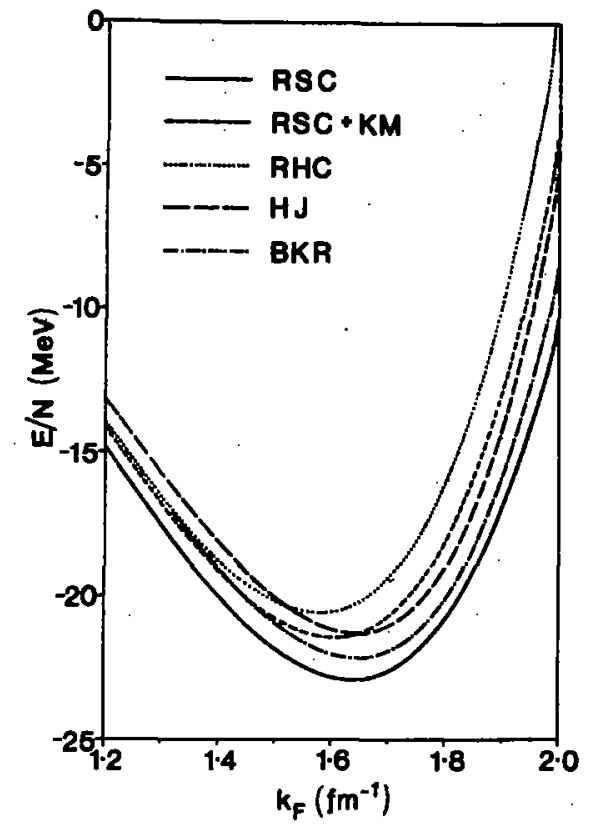

Fig. 3. The enoroy per particle of nuclear matter as a function of Fermi momentum, uaing the prewent mothod (with no interuction in states with $J_{>}$2). The potentials used are: (i) Roid soft core 5), (RSC); (ii) RSC with a correction to the ${ }^{2} \mathrm{~S}_{0}$ state by Kermode and McKerroll ") (see text), (RSC+KM); (iii) Reid hard cores), (RHC); (iv) Hamada-Johnston 7), (HJ); and

(v) Breasel-Kerman-Rouben 4), (BKR).

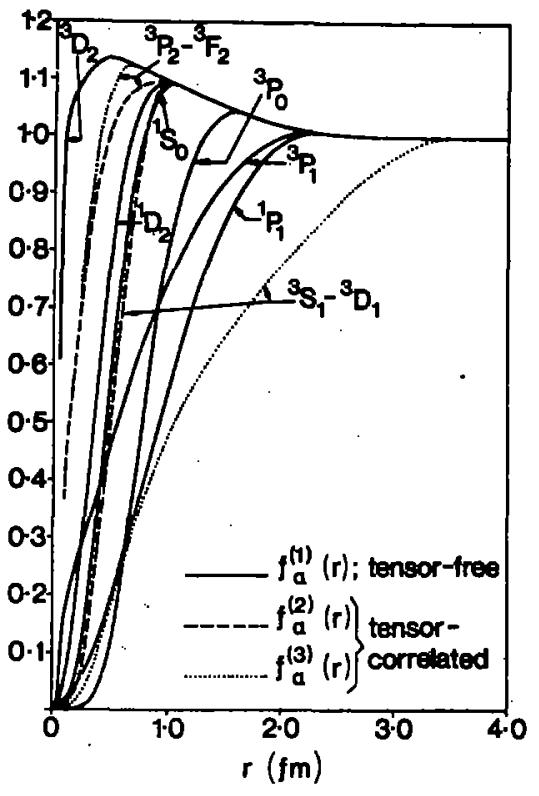

Fig. 4. The nuclear matter correlation functions obtained in the present method with the RSC potential (with no interaction in states with $J>2)$, at $k_{\mathbf{r}}=1.4 \mathrm{fm}^{-1}$.

(i.e. different independent central correlation functions in the ${ }^{3} \mathrm{P}_{2}$ and ${ }^{3} \mathrm{~F}_{2}$ states). We find that with the RSC potential, for example, at $k_{\mathrm{F}}=1.6 \mathrm{fm}^{-1}$, using neither spin-orbit nor tensor correlations gives $E / N=-22.52 \mathrm{MeV}$; using spin-orbit correlations gives $E / N=-22.55 \mathrm{MeV}$; and using tensor correlations gives $E / N=-22.81$ $\mathrm{MeV}$. This example, which is quite typical for the range of potentials, clearly suggests that it is more important to allow for tensor correlations than for spin-orbit correlations, even in the ${ }^{3} \mathbf{P}_{2} \cdot{ }^{3} \mathbf{F}_{2}$ channel; and that the neglect of explicit spin-orbit correlations is a very good approximation indeed, as already suggested in sect. 2 . It should be noted however that we have already made a considerable allowance for the full spinorbit splitting by making our correlation functions $J$-dependent.

In order to investigate the effect of the neglect of the potential in states $J>2$, we have also performed a calculation using the RSC potential in states with $J \leqq 2$ plus the one-pion exchange potential (OPEP) in states with $J>2$. In this case we use correlation functions of the form (10) and (19) for all states $J \leqq 2$ as above, and of the 
Tasu 2

Channel breakdown of the enersy per nucleon (in MoV) in nuclear matter, using the RSC potential (with no interaction in states with $J>2$ )

\begin{tabular}{|c|c|c|c|c|c|}
\hline$k=\left(f m^{-1}\right)$ & 1.2 & 1.4 & 1.6 & 1.8 & 2.0 \\
\hline${ }^{1} S_{0}$ & -13.7 & -18.4 & -23.0 & -27.0 & -29.6 \\
\hline${ }^{1} \mathbf{P}_{1}$ & 1.02 & 2.14 & 4.22 & 7.81 & 13.6 \\
\hline${ }^{1} D_{2}$ & -1.49 & -2.91 & -5.11 & -8.28 & -12.6 \\
\hline${ }^{3} \mathbf{P}_{0}$ & -2.47 & -3.75 & -5.08 & -6.18 & -6.58 \\
\hline${ }^{\mathbf{S}} \mathbf{P}_{1}$ & 5.65 & 10.4 & 17.7 & 28.2 & 42.7 \\
\hline $\left.\begin{array}{l}{ }^{3} S_{1} \\
{ }^{2} D_{1}\end{array}\right)$ & $\begin{array}{r}-15.4 \\
0.69\end{array}$ & $\begin{array}{r}-19.9 \\
1.37\end{array}$ & $\begin{array}{r}-23.2 \\
2.37\end{array}$ & $\begin{array}{r}-24.3 \\
3.68\end{array}$ & $\begin{array}{r}-22.3 \\
5.26\end{array}$ \\
\hline${ }^{*} \mathbf{D}_{2}$ & -2.58 & -4.85 & -8.12 & -12.5 & -18.0 \\
\hline $\left.\begin{array}{l}{ }^{3} \mathbf{P}_{2} \\
{ }^{3} \mathbf{F}_{2}\end{array}\right\}$ & $\begin{array}{l}-4.17 \\
-0.29\end{array}$ & $\begin{array}{l}-7.84 \\
-0.64\end{array}$ & $\begin{array}{l}-13.2 \\
-1.19\end{array}$ & $\begin{array}{l}-20.5 \\
-1.99\end{array}$ & $\begin{array}{l}-29.8 \\
-3.04\end{array}$ \\
\hline sum, $E_{2} / N$ & -32.7 & -44.3 & -54.7 & -61.1 & -60.3 \\
\hline kinetic, $E_{1} / N$ & 17.9 & 24.4 & 31.9 & 40.3 & 49.8 \\
\hline$\left(E_{1}+E_{2}\right) / N$ & -14.8 & -19.9 & -22.8 & -20.8 & -10.6 \\
\hline$E(P W) / N$ & -14.2 & -20.7 & -24.5 & -24.5 & -17.9 \\
\hline$\lambda$ & -5.53 & -128 & -26.1 & -49.1 & -87.3 \\
\hline
\end{tabular}

The row Labelled E(PW)/N gives the results of Pandharipando and Wiringa "); and the final row gives the value of the Larrance multiplier $\lambda$ (in MoV) from the present calculation.

TANu 3

Same as table 2, but using the RSC+KM potential (with no interaction in states with $J>2$ )

\begin{tabular}{|c|c|c|c|c|c|}
\hline$k_{\mathrm{F}}\left(\mathrm{fm}^{-1}\right)$ & 1.2 & 1.4 & 1.6 & 1.8 & 20 \\
\hline${ }^{1} \mathrm{~S}_{0}$ & -13.1 & -17.8 & -22.6 & -27.0 & -30.4 \\
\hline${ }^{1} \mathbf{P}_{1}$ & 1.02 & 2.17 & 4.33 & 8.19 & 14.9 \\
\hline${ }^{1} \mathbf{D}_{\mathbf{2}}$ & -1.49 & -2.91 & -5.11 & -8.28 & -12.6 \\
\hline${ }^{3} \mathbf{P}_{\mathbf{0}}$ & -2.47 & -3.75 & -5.07 & -6.09 & -6.18 \\
\hline${ }^{2} \mathbf{P}_{1}$ & 5.68 & 10.5 & 17.9 & 28.8 & 44.3 \\
\hline $\left.\begin{array}{l}{ }^{3} S_{1} \\
{ }^{3} D_{1}\end{array}\right\}$ & $\begin{array}{r}-15.4 \\
0.70\end{array}$ & $\begin{array}{r}-19.7 \\
1.39\end{array}$ & $\begin{array}{r}-22.7 \\
2.41\end{array}$ & $\begin{array}{r}-22.8 \\
3.74\end{array}$ & $\begin{array}{r}-18.2 \\
5.37\end{array}$ \\
\hline${ }^{3} \mathrm{D}_{2}$ & -2.58 & -4.85 & -8.12 & -12.5 & -18.0 \\
\hline $\left.\begin{array}{l}{ }^{3} \mathbf{P}_{2} \\
{ }^{3} \mathbf{F}_{2}\end{array}\right\}$ & $\begin{array}{l}-4.17 \\
-0.29\end{array}$ & $\begin{array}{l}-7.84 \\
-0.64\end{array}$ & $\begin{array}{l}-13.2 \\
-1.19\end{array}$ & $\begin{array}{l}-20.5 \\
-1.99\end{array}$ & $\begin{array}{l}-29.7 \\
-3.04\end{array}$ \\
\hline $\operatorname{sum}, E_{2} / N$ & -32.1 & -43.5 & -53.3 & -58.4 & -53.7 \\
\hline kinetic, $E_{1} / N$ & 17.9 & 24,4 & 31.9 & 40.3 & 49.8 \\
\hline $\begin{array}{c}\left(E_{1}+E_{2}\right) / N \\
E(\mathrm{PW}) / N\end{array}$ & -14.2 & -19.1 & -21.5 & -18.1 & -3.96 \\
\hline$\lambda$ & -5.82 & -13.9 & -29.7 & -60.3 & -121 \\
\hline
\end{tabular}


TAns 4

Samo as table 2, but uing the RHC potential (with no interaction in states with $J>2$ )

\begin{tabular}{|c|c|c|c|c|c|}
\hline$k_{\mathrm{F}}\left(\mathrm{fm}^{-1}\right)$ & 1.2 & 1.4 & 1.6 & 1.8 & 2.0 \\
\hline $\left.\begin{array}{l}{ }^{1} \mathbf{S}_{0} \\
\mathbf{P}_{1} \\
\mathbf{P}_{2} \\
\mathbf{D}_{2} \\
\mathbf{P}_{0} \\
\mathbf{P}_{1} \\
\mathbf{P}_{1} \\
\mathbf{S}_{1} \\
\mathbf{D}_{1} \\
\mathbf{D}_{2} \\
\mathbf{O}_{2} \mathbf{P}_{2} \\
\mathbf{P}_{2}\end{array}\right\}$ & $\begin{array}{c}-13.6 \\
1.52 \\
-1.61 \\
-2.92 \\
6.22 \\
-15.1 \\
0.68 \\
-2.71 \\
-4.05 \\
-0.29\end{array}$ & $\begin{array}{c}-18.3 \\
2.92 \\
-3.11 \\
-4.39 \\
11.2 \\
-19.4 \\
1.39 \\
-5.09 \\
-7.64 \\
-0.62\end{array}$ & $\begin{array}{c}-22.9 \\
5.40 \\
-5.39 \\
-5.87 \\
18.6 \\
-22.2 \\
2.54 \\
-8.50 \\
-12.9 \\
-1.13\end{array}$ & $\begin{array}{c}-26.8 \\
9.67 \\
-8.64 \\
-7.00 \\
29.2 \\
-21.9 \\
4.15 \\
-13.0 \\
-20.1 \\
-1.84\end{array}$ & $\begin{array}{c}-29.2 \\
17.0 \\
-13.0 \\
-7.22 \\
44.0 \\
-16.4 \\
6.20 \\
-18.7 \\
-29.2 \\
-2.71\end{array}$ \\
\hline $\begin{array}{c}\min _{1} E_{2} / N \\
\text { kinctic, } E_{1} / N \\
\left(E_{1}+E_{2}\right) / N \\
E(P W) / N\end{array}$ & $\begin{array}{r}-31.9 \\
17.9 \\
-14.0\end{array}$ & $\begin{array}{r}-43.1 \\
24.4 \\
-18.7\end{array}$ & $\begin{array}{r}-52.5 \\
31.9 \\
-20.6\end{array}$ & $\begin{array}{r}-56.4 \\
40.3 \\
-16.0\end{array}$ & $\begin{array}{r}-49.3 \\
49.8 \\
0.47\end{array}$ \\
\hline$\lambda \lambda$ & -5.66 & -13.8 & -30.5 & -626 & -123 \\
\hline
\end{tabular}

Tame 5

Same as table 2, but using the HJ potential (with no interaction in states with $J>2$ )

\begin{tabular}{|c|c|c|c|c|c|}
\hline$k_{5}\left(\mathrm{fm}^{-1}\right)$ & 1.2 & 1.4 & 1.6 & 1.8 & 2.0 \\
\hline $\left.\begin{array}{l}{ }^{1} \mathbf{S}_{0} \\
{ }^{1} \mathbf{P}_{1} \\
{ }^{1} \mathbf{D}_{2} \\
{ }^{3} \mathbf{P}_{0} \\
{ }^{1} \mathbf{P}_{1} \\
{ }^{3} \mathbf{S}_{1} \\
\mathbf{D}_{1} \\
\mathbf{D}_{1} \mathbf{D}_{2} \\
\mathbf{D}^{3} \mathbf{P}_{2} \\
{ }^{3} \mathbf{F}_{2}\end{array}\right\}$ & $\begin{array}{c}-13.5 \\
1.93 \\
-1.56 \\
-2.94 \\
5.35 \\
-15.7 \\
0.61 \\
-2.33 \\
-2.54 \\
-0.37\end{array}$ & $\begin{array}{r}-18.2 \\
3.30 \\
-3.06 \\
-4.60 \\
9.38 \\
-20.2 \\
1.25 \\
-4.39 \\
-\quad 5.05 \\
-0.83\end{array}$ & $\begin{array}{r}-22.9 \\
5.40 \\
-5.39 \\
-6.48 \\
-15.3 \\
-23.3 \\
-2.30 \\
-7.36 \\
-8.94 \\
-1.63\end{array}$ & $\begin{array}{c}-27.0 \\
8.70 \\
-8.77 \\
-8.29 \\
23.8 \\
-22.9 \\
3.85 \\
-11.4 \\
-14.5 \\
-\quad-2.87\end{array}$ & $\begin{array}{r}-29.4 \\
14.1 \\
-13.4 \\
-9.50 \\
36.2 \\
-15.9 \\
-5.92 \\
-16.4 \\
-22.0 \\
-4.63\end{array}$ \\
\hline $\begin{array}{c}\operatorname{sim}, E_{2} / N \\
\text { kinetic, } E_{1} / N \\
\left(E_{1}+E_{2}\right) / N \\
E(\mathrm{PW}) / N\end{array}$ & $\begin{array}{r}-31.1 \\
17.9 \\
-13.1 \\
-11.5\end{array}$ & $\begin{array}{r}-42.5 \\
24.4 \\
-18.1 \\
-16.2\end{array}$ & $\begin{array}{r}-53.0 \\
31.9 \\
-21.2 \\
-18.8\end{array}$ & $\begin{array}{r}-59.3 \\
40.3 \\
-19.0 \\
-17.6\end{array}$ & $\begin{array}{r}-55.0 \\
49.8 \\
-5.24 \\
-9.1\end{array}$ \\
\hline$\lambda$ & -4.93 & -12.2 & -28.4 & -63.8 & -140 \\
\hline
\end{tabular}

form (35) for all states $J>2$. In fig. 5 we show the contribution due to the OPEP part of the potential as a function of Fermi momentum. Although in principle there is a coupling of the energy in high- $J$ states with that in low- $J$ states through the integral constraint (27) [i.e. the same Lagrange multiplier $\lambda$ appears in each of eqs. (30)-(31)], we find in practice that this coupling of the high- $J$ states is weak and that the energy for states $J>2$ may simply be added to that for states with $J \leq 2$ evaluated assuming no interaction in the $J>2$ states. For example, for the $R S C$ potential at $k_{\mathrm{F}}=1.6$ 
TABrs 6

Samo as table 2, but wing the BKR potential (with no interaction in states with $J>2$ )

\begin{tabular}{|c|c|c|c|c|c|}
\hline$k_{\mathbf{p}}\left(\mathrm{fm}^{-1}\right)$ & 1.2 & 1.4 & 1.6 & 1.8 & 20 \\
\hline $\begin{array}{l}{ }^{1} S_{0} \\
{ }^{1} \mathbf{P}_{1} \\
{ }^{1} D_{2} \\
{ }^{3} \mathbf{P}_{0} \\
{ }^{3} \mathbf{P}_{1} \\
{ }^{3} \mathbf{S}_{1} \\
{ }^{3} \mathbf{D}_{1} \\
{ }^{3} \mathbf{D}_{2} \\
{ }^{3} \mathbf{P}_{2} \\
{ }^{3} \mathbf{F}_{2}\end{array}$ & $\begin{array}{r}-13.7 \\
1.96 \\
-1.71 \\
-3.26 \\
5.52 \\
-15.9 \\
0.68 \\
-2.45 \\
-2.51 \\
-0.42\end{array}$ & $\begin{array}{r}-18.4 \\
3.36 \\
-3.33 \\
-5.11 \\
9.73 \\
-20.5 \\
1.32 \\
-4.59 \\
-4.84 \\
-0.95\end{array}$ & $\begin{array}{r}-23.0 \\
5.46 \\
-5.81 \\
-7.22 \\
15.8 \\
-23.7 \\
2.40 \\
-7.67 \\
-\quad 8.30 \\
-\quad 1.85\end{array}$ & $\begin{array}{c}-26.9 \\
8.70 \\
-9.38 \\
-9.34 \\
24.5 \\
-23.9 \\
-3.97 \\
-11.8 \\
-13.0 \\
-3.25\end{array}$ & $\begin{array}{r}-29.3 \\
13.6 \\
-14.2 \\
-11.1 \\
36.7 \\
-19.0 \\
6.06 \\
-17.0 \\
-19.0 \\
-5.25\end{array}$ \\
\hline $\begin{array}{c}\operatorname{sum}, E_{2} / N \\
\text { kinetic, } E_{1} / N \\
\left(E_{1}+E_{2}\right) / N \\
E(\mathrm{PW}) / N\end{array}$ & $\begin{array}{r}-31.8 \\
-17.9 \\
-13.8 \\
-13.4\end{array}$ & $\begin{array}{r}-43.3 \\
24.4 \\
-19.0 \\
-19.4\end{array}$ & $\begin{array}{r}-53.9 \\
31.9 \\
-22.0 \\
-23.6\end{array}$ & $\begin{array}{r}-60.4 \\
40.3 \\
-20.1 \\
-24.7\end{array}$ & $\begin{array}{r}-58.4 \\
49.8 \\
-8.6 \\
-20.2\end{array}$ \\
\hline$\lambda$ & -4.15 & -11.9 & -26.9 & -55.8 & -103 \\
\hline
\end{tabular}

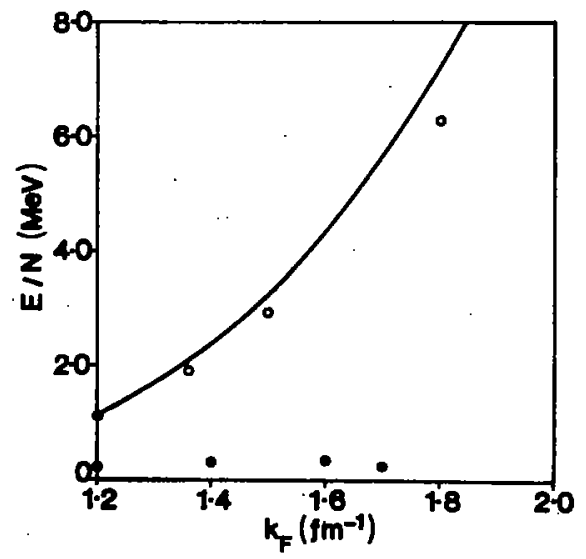

Fig. 5. The contribution to the energy per particle of nuclear matter from OPEP in states with $J>2$, from the present calculation (solid curve) and the calculation of Haftel and Tabakin ${ }^{11}$ ) (open circles). The results of Sprung, reported by Bethe ${ }^{12}$ ), using the phase-shift approximation in states with $3 \leqq J \leqq 5$ and OPEP in states with $J>5$, are also shown (solid circlos). In all cases tho RSC potential is usod in states with $J \leqq 2$. Seo text for dotails of the calculations.

$\mathrm{fm}^{-1}$, we find $E(J \leqq 2) / N=-22.81 \mathrm{MeV}$ when evaluated with no interaction in states with $J>2$, and $E(J \leqq 2) / N=-22.62 \mathrm{MeV}$ when ovaluated with OPEP in states $J>2$. By way of comparison, we also show the OPEP results of Haftel and Tabakin ${ }^{11}$ ). These authors have performed a Brueckner $G$-matrix calculation for nuclear matter with the RSC potential for states $J \leqq 2$ and OPEP in states $J \geq 3$ (up to and including all $L=7$ states). There is, however, no reason to believe that OPEP 
is a particularly good representation of the potential for states with $J>2$, and we have preferred not to include such states in our results. We also show in fig. 4 another estimate of the contribution from states with $J>2$ to the energy per particle in nuclear matter due to Sprung and reported by Bethe ${ }^{12}$ ). Sprung, in the context of his Brueckner $G$-matrix calculation, also uses the RSC potential for states with $J<2$, but for states with $3 \leqq J \leqq 5$ he uses the phase-shift approximation and the experimental phase-shifts, and again OPEP for states with $J>5$.

At this stage it is worth pointing out that although at the densities considered the high-J states do effectively decouple from the low- $J$ states (in the sense, indicated above, that their contribution to the energy is to a good approximation additive), this is not true for the states which make an important contribution to the energy, or more exactly to the constraint integral $k$ of eqs. (27) or (29). An excellent example of this is provided by two of the potentials considered here, namely RSC and RSC+KM. Thus, although these potentials differ only in the ${ }^{1} \mathrm{~S}_{0}$ channel, the difference in total energy per particle for the two potentials does not by any means arise from just this channel, as can clearly be seen from tables 2 and 3 . Thus, although at a density corresponding to $k_{F}=1.2 \mathrm{fm}^{-1}$, the $\mathrm{RSC}+\mathrm{KM}$ potential gives $0.6 \mathrm{MeV}$ per particle less binding than the $R S C$ potential which comes almost exclusively from the ${ }^{1} S_{0}$ channel, the difference is not wholly explained by this channel alone as the density is increased. Thus, at $k_{\mathrm{F}}=1.6 \mathrm{fm}^{-1}$, the RSC+KM potential gives $1.3 \mathrm{MeV}$ per particle less binding than the $R S C$ potential, of which only $0.4 \mathrm{MeV}$ arises from the ${ }^{1} \mathrm{~S}_{0}$ channel; a further $0.5 \mathrm{MeV}$ now arising from the most strongly coupled channel, ${ }^{3} \mathrm{~S}_{1} \cdot{ }^{3} \mathrm{D}_{1}$, and the remainder from the $\mathrm{P}_{1}$ states. Even more dramatically, at $k_{F}=2.0 \mathrm{fm}^{-1}$, the RSC+KM potential gives 6:6 MeV per particle less binding than RSC, whereas in this case the ${ }^{1} \mathrm{~S}_{0}$ channel actually gives $0.8 \mathrm{MeV}$ per particle more binding, whilst the ${ }^{3} \mathrm{~S}_{1}{ }^{3} \mathrm{D}_{1}$ channel gives $4.2 \mathrm{MeV}$ per particle less binding, and the remainder again comes principally from the $P_{1}$ states. The correlation of these observations with the

\section{TAre 7}

Channol breakdown of the contributions to the normalisation integral $k$ of eq. (29) from thovarious orthosonal projections of the wave function, for nuclear matter using the RSC potential (with no interaction in states with $J>2$ )

\begin{tabular}{|c|c|c|c|c|c|}
\hline$k_{F}\left(\mathrm{fm}^{-1}\right)$ & 1.2 & 1.4 & 1.6 & 1.8 & 2.0 \\
\hline${ }^{1} S_{0}$ & 0.07 & 0.10 & 0.15 & 0.19 & 0.22 \\
\hline${ }^{1} \mathbf{P}_{1}$ & 0.06 & 0.08 & 0.09 & 0.09 & 0.08 \\
\hline${ }^{1} \mathbf{D}_{2}$ & 0.00 & 0.00 & 0.00 & 0.00 & 0.00 \\
\hline${ }^{3} \mathbf{P}_{0}$ & 0.01 & 0.02 & 0.03 & 0.04 & 0.05 \\
\hline${ }^{3} \mathbf{P}_{1}$ & 0.18 & 0.16 & 0.13 & 0.10 & 0.07 \\
\hline $\operatorname{sS}_{1}=D_{1}$ & 0.05 & 0.07 & 0.10 & 0.13 & 0.16 \\
\hline$S_{1}=D_{1}$ & 0.62 & 0.57 & 0.50 & 0.44 & 0.40 \\
\hline${ }^{3} \mathbf{D}_{2}$ & 0.00 & 0.00 & 0.00 & 0.00 & 0.00 \\
\hline${ }^{3} P_{2}-{ }^{3} F_{2}\left\{f^{(2)} Q\right.$ & 0.00 & 0.00 & 0.00 & 0.00 & 0.00 \\
\hline$(1-Q)$ & 0.00 & 0.00 & 0.00 & 0.00 & 0.00 \\
\hline
\end{tabular}


varying degree of contribution to the constraint (or normalization) integral $k$ of eq. (29) from the various channels, as a function of density, shown in table 7 for the RSC potential, is clear.

We now turn to a comparison of our nuclear matter results with those recently reported by Pandharipande and Wiringa " (hereafter referred to as PW), who have also used three of the potentials employed here, namely RSC, HJ and BKR. In their variational method, PW first evaluate the two-body energy by defining a healing distance, $d_{0}$, for their correlation functions, and then solving a Schrodinger-like equation for the correlation functions for the range $0 \leqq r \leqq d_{0}$. They add in a constant state-dependent potential $\lambda_{2}$ for each channel $\alpha$, in order to satisfy the necessary boundary conditions of the continuity of the correlation functions and their first derivatives at the healing radius $d_{0}$. In terms of the correlation functions so defined, and after a certain amount of state-averaging, they further evaluate the sum of what they hope to be all of the significant many-body cluster contributions (MBCC) to the energy. Before making a direct comparison between the results of the present work and those of PW, we make the following three remarks.

First, PW do not assume zero interaction in states with $J>2$ as we do, but instead use an effective potential derived from the $D$-states for even-parity states with $L \geq 4$, and from the F-states for odd-parity states with $L \geq 5$. It is by no means clear that this is a better prescription than using, for example, OPEP in states with $J \geqq 3$, or even than using zero interaction in these states. However, since in the scheme of PW it is necessary to define an average effective potential depending only on the total spin and isospin of the two-body channels, for the evaluation of the MBCC to the energy, such a treatment of the higher partial waves is natural in their calculations. Crudely, one might expect that the different treatment of the higher channels by $\mathrm{PW}$ will have an effect of certainly not more than that obtained by introducing OPEP for states with $J \geqq 3$, given in fig. 5 .

Secondly, in their evaluation of the two-body cluster contribution to the energy, PW allow their correlation functions to depend on the relative momentum, $k$, of the interacting pair; although in evaluating the MBCC this momentum dependence is suitably averaged. For simplicity, we have excluded this momentum dependence, although it should be noted that we have summed over the relative momentum states before minimising, to obtain our momentum-independent correlation functions, which is clearly a better procedure than, for example, choosing an average momentum to define the correlation function. Considering the very similar form of the correlation functions obtained by PW for very different relative momenta [and see fig. 9 of ref. $\left.{ }^{8}\right)$ ], we believe that this is a very good approximation indeed; and that the introduction of momentum dependence into our correlation functions would not significantly lower our energies, although the difficulty of their evaluation would be greatly increased by so doing.

Thirdly, PW allow for the effect of increasing $d_{0}$ from their standard value of $2 r_{0}$, where $r_{0}=\left(\frac{4}{3} \pi \rho\right)^{-t}$ is the unit radius. They find that by so doing, with the Reid 
potential at $k_{\mathrm{F}}=1.6 \mathrm{fm}^{-1}$ as an example, they are able to lower the energy (including MBCC), apparently without bound. This is certainly a disturbing feature of the PW calculations, and it indicates either that by increasing $d_{0}$ they could significantly lower all of their energies, or that for this density and with $d_{0} \geqslant 2 r_{0}$ they have not in fact succeeded in evaluating all of the significant MBCC. Presumably this in turn means that the energies evaluated with $d_{0}=2 r_{0}$ must also be regarded as being rather uncertain.

In tables 2, 5 and 6, we give for comparison, in the rows labelled $E(\mathrm{PW}) / N$ the final energies per particle obtained by PW including their MBCC. We refer the reader to ref. $^{8}$ ) for a more detailed channel breakdown and an analysis of the various MBCC to these energies. For both the RSC and BKR potentials, our results are in good agreement with those of $\mathrm{PW}$ for $k_{\mathrm{F}} \lessgtr 1.6 \mathrm{fm}^{-1}$, and become less bound thereafter with increasing density. For the HJ potential the agreement remains good up to beyond $k_{\mathrm{F}}=1.8 \mathrm{fm}^{-1}$, but in this case we obtain slightly more binding than $\mathrm{PW}$. Wo expect that if allowance is made for our different treatment of states with $J \geqq 3$, as explained above, then the agreement for the HJ potential would be improved, whilst that for the RSC and BKR potentials would be slightly worsened. Nevertheless, it is extremely encouraging that for $k_{\mathrm{F}} \leqslant 1.6 \mathrm{fm}^{-1}$, our results differ from those of $\mathrm{PW}$ by not more than about $2 \mathrm{MeV}$ (i.e. by not more than about $10 \%$ ) in all cases. With tho possible exception of the BKR potential it seems that our saturation densities also lie very close to those found by $\mathrm{PW}$.

Each of the five potentials considered here gives a value for the binding energy per particle of nuclear matter at equilibrium of over $20 \mathrm{MeV}$ at a density corresponding to $k_{F} \approx 1.6-1.7 \mathrm{fm}^{-1}$, in constrast to the empirical values of about $16 \mathrm{MoV}$ at $k_{\mathrm{F}} \approx 1.4 \mathrm{fm}^{-1}$. It is therefore encouraging that improving the fit of the potential to the phase-shift data and allowing for different np and pp potentials in the ${ }^{1} \mathrm{~S}_{0}$ state (i.e. introducing the Kermode-McKerrell correction to the RSC potential) both reduces the binding energy and the saturation density, although this is clearly a small effect. The precise manner by which this occurs is also rather intricate, as previously mentioned. As expected, we also notice that the hard-core potentials give consistently less binding than the soft-core potentials.

\section{Discuselon}

We have previously performed calculations on a number of central potentials using the LOCV method outlined here ${ }^{1,10,13}$ ), and we have been able to compare our results with what are probably good approximations to the exact many-body energies for these potentials. For both Fermi ${ }^{1,13}$ ) and Bose ${ }^{10}$ ) systems we havo found that there are two types of potentials for which our LOCV method gives consistently good results. The first type, typified by the $\operatorname{RSC}^{1} \mathrm{~S}_{0}$ potential (acting in all channels), comprises potentials with a strong short-range repulsion followed by a strong attraction. In this case we find that for densities considerably higher than that of nuclear matter, 
the strong attraction of the potential forces the correlation function to heal rapidly to our assumed long-range form, and our integral constraint (27) is satisfied automatically as an inequality. In these cases, the only constraint operating on the correlation function is the boundedness relation (26), which restricts its overshoot. Presumably in these cases the cluster expansion for the exact two-body radial distribution function, $g(r)$, is automatically rapidly convergent and, provided that the lowest order energy has been efficiently minimised, the replacement of $g(r)$ by its lowest order approximant,

$$
g^{(2)}(r)=f^{2}(r) \phi^{2}(r)
$$

where $\phi(r)$ is the uncorrelated two-body wave function, is then a very good approximation.

The second type of potential for which our LOCV method again gives excellent results, both for fermions ${ }^{13}$ ) and bosons ${ }^{10}$ ), comprises purely repulsive models, such as the so-called Bethe "homework problem" potential (i.e. the repulsive part only of the RSC ${ }^{1} S_{0}$ potential acting in all channels). In these cases, however, the integral constraint (27) plays a crucial role, at all densities, in forcing the correlation function to heal and in restricting the size of the wound in the correlated two-body wave function. Our understanding of the good agreement for these potentials is as follows. The exact expectation value $\langle V\rangle$ of the potential energy per particle may be written as,

$$
\langle V\rangle=\frac{1}{2} \rho \int \mathrm{d} r_{12} V\left(r_{12}\right) \theta\left(r_{12}\right),
$$

where $g\left(r_{12}\right)$ is again the exact two-body distribution function, which must satisfy the normalisation condition,

$$
\rho \int \mathrm{d} r_{12}\left[1-g\left(r_{12}\right)\right]=1 .
$$

Since this normalisation condition is also imposed on our lowest order approximation $g^{(2)}(r)$ to $g(r)$ [i.e., when the equality sign is taken in eq. (27), or equivalently when $\lambda$ $\neq 0$ ], then provided that $V(r)$ is not changing rapidly in the region where $g(r)$ is not well approximated by $g^{(2)}(\boldsymbol{r})$, it should still be a good approximation to evaluate $\langle\boldsymbol{V}\rangle$ from eq. (42) with this approximation for $g(r)$. Of course, the restriction that our $g^{(2)}(r)$ should satisfy the normalisation condition could represent an unreasonable restriction on our variational space, but in practice this appears not to be the case.

Conversely, the potentials for which our LOCV method (in common with many other approximations) does not seem to be so reliable, are those potentials with a longranged repulsive core followed by a weak attractive component, such as the central part of the $\operatorname{RSC}^{3} \mathrm{~S}_{1} \cdot{ }^{3} \mathrm{D}_{1}$ potential acting in all partial waves. In such cases $g^{(2)}(r)$ is not a good approximation to $g(r)$ for a large region of space and neither is $V(r)$ slowly varying in this region.

The realistic potentials considered in the present work are of a rather complicated nature, and in particular are quite different in the various $\alpha \equiv(J S T)$ channels. For 
those states, such as the ${ }^{1} S_{0}$ state, with a potential which, when used in all states, generates a function $g^{(2)}(r)$ which approximates well to the exact $g(r)$, it should still be a good approximation to replace $g_{a}(r)$ by $g_{a}^{(2)}(r)$. This should be true for all the $L=0$ channels (clearly the most important), except for that associated with the $f^{(3)}(1-Q)$ projector in the coupled ${ }^{3} \mathrm{~S}_{1}{ }^{3} \mathrm{D}_{1}$ states. This channel, however, has an associated effective potential [see eq. (31)] of $V^{c}-4 V^{\mathrm{T}}-2 V^{L S}$, which is wholly repulsive for each of the five potentials considered here. Provided that a large proportion of the normalisation integral $k$ of eq. (29) derives from this channel, both for our $g^{(2)}(r)$ and for the actual $g(r)$, the reasoning applied to purely repulsive central potentials above should still apply, and we again hope to obtain a reasonable estimate of the energy. In table 7 we have shown a channel-by-channel breakdown of the contributions to the normalisation integral $k$, using the $\mathrm{RSC}$ potential as illustration. For $k_{\mathrm{F}} \leqslant 1.6 \mathrm{fm}^{-1}$, we see that over half of the total contribution does indeed derive from this purely repulsive channel; although of course we have no means of checking whether this is also the case for the exact $g(r)$. Above $k_{\mathrm{F}} \approx 1.6 \mathrm{fm}^{-1}$, the contributions to $k$ from the other $L=0$ states become appreciable, and the interaction among the various channels induced by our normalisation constraint becomes considerable, as already discussed under a different guise in sect. 4. Such simple arguments as the above can then no longer be applied.

Our previous calculations with the central part of the $\operatorname{RSC}^{3} \mathrm{~S}_{1}-{ }^{3} \mathrm{D}_{1}$ potential, acting in all states; [potential V2 of ref. ${ }^{1}$ )] have suggested that this is a very difficult potential to handle in the context of our LOCV method (and probably in most other methods as well) for the reasons already cited. However, by the addition of the tensor force and the inclusion of tensor correlations, the effective potential in the $f^{(2)} Q$

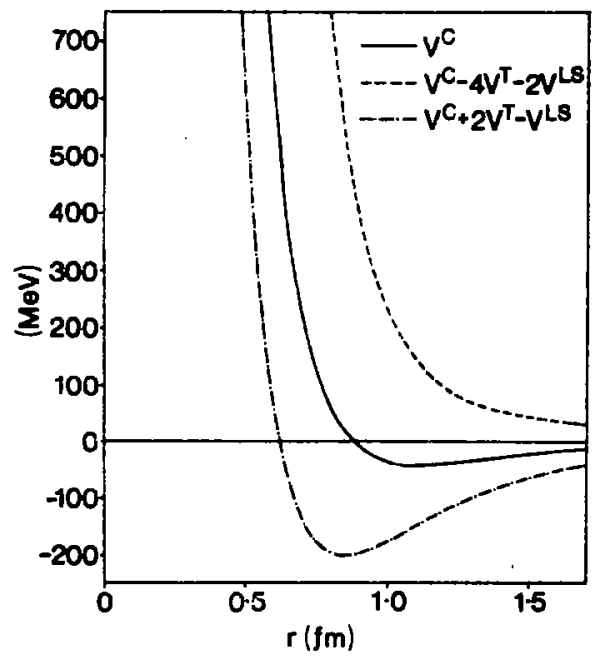

Fig. 6. Three aspects of the RSC potential in the ${ }^{2} S_{1}-D_{1}$ channel, $V(r)=V^{c}(r)+V^{T}(r) S_{12}+$ $V^{L}(r) L \cdot S$. 
projection of the ${ }^{3} S_{1}-{ }^{3} D_{1}$ channel becomes $V^{C}+2 V^{T}-V^{L S}$, which is very much more attractive than the central part $V^{C}$ alone; whilst the effective potential in the orthogonal $f^{(3)}(1-Q)$ projection becomes $V^{c}-4 V^{T}-2 V^{L S}$, which is everywhere repulsive. These statements are illustrated in fig. 6 . It is interesting to note that it may thus be the inclusion of the tensor (and spin-orbit) forces, and the correct incorporation of tensor correlations, which allows us to deal accurately with the contributions to the energy of nuclear matter from all of the states of realistic potentials, and to obtain such good agreement with the far more ambitious and complicated calculations of PW.

Whether or not the preceding arguments are quantitatively correct, we still have to face the fact that for $k_{\mathrm{F}} \$ 1.6 \mathrm{fm}^{-1}$, our nuclear matter results agree extremely well with those of $\mathrm{PW}$, which are hopefully reliable upper bounds on the energy up to this density, and yet differ considerably from the accepted experimental equilibrium values of about $-16 \mathrm{MeV}$ per particle at $k_{\mathrm{F}} \approx 1.4 \mathrm{fm}^{-1}$. Before reaching the conclusion that none of the five commonly accepted potentials used here is in fact a realistic model of the NN force, other possible reasons for this discrepancy should be studied. We conclude with some brief remarks concerning one such possible explanation, namely the neglect of the internal degrees of freedom of the nucleons that permit them to excite to higher resonances in intermediate states.

Several authors ${ }^{14,15}$ ) have published estimates for the effect on the energy of nuclear matter if one specifically allows the nucleons to excite to $\Delta(1236)$ resonances, and reach the conclusion that this has the effect of weakening the effective ${ }^{1} S_{0}$ interaction in the many-body medium. Whilst it is clearly inconsistent to include only one of many possible such higher-order processes, it is interesting to do so in this case in order to gain a qualitative idea of the size of the effects that we might expect. Green and Niskanen ${ }^{14}$ ) fit their estimates for the change in total energy from explicitly allowing $\Delta(1236)$ resonances in nuclear matter, according to the formula $\delta E / N=$ $0.71\left(k_{\mathrm{F}} \times 1 \mathrm{fm}\right)^{3.87} \mathrm{MeV}$. If, for amusement, we add this energy to our results for, say, the RSC potential, we find saturation at $k_{\mathrm{F}}=1.40 \mathrm{fm}^{-1}$ with an energy per particle of $-14.8 \mathrm{MeV}$. On the other hand, Day and Coester ${ }^{15}$ ) give an estimate for the effect of allowing the $\Delta(1236)$ resonance to couple to both the S- and P-channeis, and fit their results approximately to the formula $\delta E / N=1.02 k_{\mathrm{F}}^{3.76}+0.299 k_{\mathrm{F}}^{3.21}$ $\mathrm{MeV}$, with $k_{\mathrm{F}}$ in units of $\mathrm{fm}^{-1}$. Again, added to our results for the $\mathrm{RSC}$ potential, this estimate gives saturation at $k_{\mathrm{F}}=1.45 \mathrm{fm}^{-1}$ with an energy per particle of $-14.8 \mathrm{MeV}$. We stress again that these results should not be taken too seriously, but they do serve to show that the inclusion of at least one of these higher-order processes brings our results into the experimental arena.

In this context we remark finally that the LOCV method described in this work gives much better agreement with the variational calculations of $P W$ which include MBCC, than either lowest order Brueckner theory or the LOCV method of Pandharipande. We hope therefore that tho LOCV method discussed above will prove, because of its great simplicity, to be a useful tool in the study of the possible higher-order processes in nuclear matter. Such a study is now clearly needed. 


\section{References}

1) J. C. Owen, R. F. Bishop and J. M. Irvino, Nucl. Phys. A274 (1976) 108

2) M. L. Rintig, W. J. Tor Louw and J. W. Clark, Phyi. Rov. CS (1972) 695; C3 (1971) 1504

3) J. L. Gammel, R. S. Christian and R. M. Thaler, Phys. Rev. 105 (1957) 311;

J. L. Gammol and R. M. Thaler, in Progress in olementary particle and cosmic ray phyaica, vol. 5, od. J. G. Wilson and S. A. Wouthuysen (North-Holland, Amsterdam, 1960)

4) C. N. Breseel, A. K. Kerman and B. Rouben, Nucl. Phys. A124 (1969) 624

5) R. V. Roid, Ann. of Phys. 50 (1968) 411

6) M. W. Kermodo and A. MaKerrell, J. Phys. G1 (1975) 623

7) T. Hamada and I. D. Johnston, Nucl. Phyt. 34 (1962) 382

8) V. R. Pandharipando and R. B. Wiringe, Nucl. Phys. A266 (1976) 269

9) J. C. Owen, R. F. Bishop and J. M. Irvine, Phys, Lett 61B (1976) 147

10) J. C. Owen, R. F. Bishop and J. M. Irvino, Ann. of Phys. to bo published

11) M. I. Haftel and F. Tabakio, Nucl. Phys A158 (1970) 1

12) H. A. Bethe, Ann. Rev. Nucl. Sci. 21 (1971) 93;

P. K. Banerjoe and D. W. L. Sprung, Can. J. Phys. 49 (1971) 1899

13) J. C. Owen, R. F. Bishop and J. M. Irvine, Phys. Lett., to be published

14) A. M. Green and J. A. Ninkanen, Nucl. Phys. A249 (1973) 493

15) B. D. Day and F. Coester, Phyn. Rev. C4 (1976) 1720 